\title{
Avaliação de adsorventes sólidos para determinação de pesticidas organoclorados e bifenilos policlorados na atmosfera marinha
}

\author{
Rosalinda Carmela MONTONE \& Rolf Roland WEBER
}

Instituto Oceanográfico da Universidade de Sāo Paulo

\begin{abstract}
(Caixa Postal 9075, 01051 São Paulo, SP)
150.
\end{abstract}
- Abstract: A sampling system was developed to be used in coastal areas and on board of oceanographic vessels for the determination of chlorinated pesticides and polychlorinated biphenyls (PCB) in the atmosphere. The basis of the system is the pre-concentration step on solid adsorbents. Florisil, XAD-2 resin and polyurethane foam (PUF) were tested. The sampling efficiency presented variations between 16-118\% (florisil), 41$131 \%$ (XAD-2) and $66-122 \%$ (PUF) depending on the organochlorine compound.

- Descriptors: Organochlorinated pesticides, Adsorption, PCB, Air pollution, Air sampling, Air-sea interaction.

- Descritores: Pesticidas organoclorados, Adsorção, PCB, Poluição do ar, Amostragem do ar, Interação ar-mar.

\section{Introdução}

Antes de 1950, as determinaçōes de compostos organoclorados envolviam métodos nāo específicos que determinavam apenas o teor de cloro total da espécie. Durante a década de 50 , métodos colorimétricos foram desenvolvidos, porém, a sensibilidade ainda era, apenas, da ordem de partes por milhão. Somente com o desenvolvimento de métodos analíticos mais específicos e sensíveis, como as técnicas cromatográficas, espectroscopias de infravermelho e de massa, na década de 60 , é que se tornou viável a determinaçāo quantitativa dos organoclorados em amostras ambientais, isto é, abaixo de partes por bilhäo (ppb).

As primeiras determinaçōes em ambientes marinhos eram relativas principalmente a níveis de organoclorados em organismos e sedimentos marinhos, especialmente de áreas costeiras. Mais tarde, ficou demonstrado que o principal meio de transporte destes compostos para os oceanos era a atmosfera (Woodwell et al., 1971).

As determinaçōes de organoclorados na atmosfera são relativamente recentes, pois, a coleta destes compostos apresentam dificuldades consideráveis. Como os níveis são da ordem de $\mathrm{ng} / \mathrm{m}^{3}$ a análise direta é inviável e necessita-se de grandes volumes de ar para sua deteção.

$\overline{\text { Contr. } n^{2} 725}$ do Inst. oceanogr. da Usp.
Faz-se, entāo, necessário a utilização de adsorventes adequados para as técnicas de pré-concentração.

Os organoclorados presentes na atmosfera estão na forma particulada ou de vapor e ambas as formas coexistem (Wheatley, 1973). A maior parte, porém, está quase que exclusivamente na fase de vapor com somente uma pequena fraçāo associada às particulas de aerosol (Atlas \& Giam, 1986).

Somente em meados dos anos 70 , com o desenvolvimento de coletores usando adsorventes sólidos é que foi possível amostrar grandes volumes de ar para a determinação de poluentes orgânicos tanto na fase de vapor como na forma particulada. Assim, os organoclorados e outros contaminantes orgânicos da atmosfera foram encontrados no Atlântico Norte (Bidleman \& Olney, 1974; Rice et al., 1977; Giam et al., 1978 e Bidleman et al., 1981), no Golfo do México (Giam et al., 1978, 1980), nas Illhas Britânicas (Dawson \& Riley, 1977), no Golfo Pérsico (Bidleman \& Leonard, 1982), nos oceanos Índico e Pacífico (Tanabe \& Tatsukawa, 1980; 1983) e no Oceano Antártico (Tanabe et al., 1980a,b).

Van Dyk \& Visweswarian (1975) discutiram os vários métodos de amostragens no ar. Revisōes dos métodos de coleta, tipos e concentraçōes de orgânicos no ar, incluindo os organoclorados foram feitas por Lewis (1976), Lamb et al. (1980), Simoneit \& Mazureck (1981) e Bidleman (1985). A Agência de Proteção Ambiental dos Estados Unidos (EPA, 1980) e Riggin (1984) também publicaram a descriçāo da amostragem e métodos analíticos para pesticidas no ar. Os adsorventes sólidos mais usados para 
coleta de organoclorados são as resinas poliméricas (XAD-2, XAD-4, Tenax, Ambersorb, Chromosorb e Porapak), florisil e espuma de poliuretano.

O objetivo deste trabalho foi a avaliação da eficiência de três adsorventes sólidos (resina XAD-2, florisil e espuma de poliuretano), utilizados na pré-concentraçẳo de pesticidas organoclorados e bifenilos policlorados (PCBs) na atmosfera.

\section{Parte experimental}

Foram testados os seguintes adsorventes: florisil (Fisher Scient. Co), resina XAD-2 (Rhom Haas) e espuma de poliuretano comercial com densidade $0,022 \mathrm{~g} / \mathrm{cm}^{3}$ e diâmetro de $1,5 \mathrm{~cm}$ por $7,8 \mathrm{~cm}$ de comprimento.

A purificaçāo destes adsorventes está descrita em Montone (1987). Toda a vidraria e solventes utilizados foram testados e apresentaram valores de brancos aceitáveis, ou seja, os picos cromatográficos não excederam três vezes o ruido.

Os adsorventes florisil, resina XAD-2 e espuma de poliuretano (EPU) foram avaliados quanto a sua eficiência para coletar e reter os compostos organoclorados usando-se um amostrador (Fig. 1) que compreende uma bomba de vácuo, um medidor de vazão, dois cartuchos de vidro com placa sinterizada e um frasco gerador.

Os adsorventes foram colocados em dois cartuchos de vidro e, a seguir, $1 \mathrm{ml}$ de uma quantidade conhecida de padrōes foi adicionada ao frasco gerador conectado ao cartucho de vidro. Uma bomba de ar, tipo compressor-aspirador, foi conectada na extremidade esquerda do amostrador, bombeando-se $1 \mathrm{~m}^{3} \mathrm{de}$ ar com uma vazão de $15 \mathrm{l} / \mathrm{min}$, através dos adsorventes.
A eficiência de coleta (EC) é definida como:

$\% E C=m_{1} /\left(m_{0}-m_{r}\right) \cdot 100$

onde:

$\mathbf{m}_{\mathbf{0}}=$ é a quantidade inicial de organoclorado adicionado no frasco gerador;

$\mathbf{m}_{1}$ = é a quantidade de organoclorado encontrado no primeiro plug;

$\mathbf{m}_{\mathbf{r}}=$ é a quantidade residual de organoclorado encontrado no frasco gerador, após o teste.

A quantidade de organoclorado encontrado no segundo plug, $\mathrm{m}_{2}$, foi usada para determinar a porcentagem total da eficiência de coleta. Todas as quantidades foram corrigidas para os valores de branco.

Este teste foi baseado no método utilizado por Lewis \& MacLeod (1982).

A resina XAD-2 (6 g) e a espuma de poliuretano $(0,30$ g) foram extraídas com $125 \mathrm{ml}$ de $\mathrm{n}$-hexano grau pesticida (Merck 4371) num Extrator Soxhlet, por 4 horas. O florisil $(10 \mathrm{~g})$ foi eluído com $80 \mathrm{ml}$ de etanol bidestilado e extraído duas vezes com $30 \mathrm{ml}$ de $\mathrm{n}$-hexano e $10 \mathrm{ml}$ de água livre de orgânicos.

O procedimento analítico para a determinação dos organoclorados está descrito em Montone (1987).

Os organoclorados foram identificados por comparaçāo dos tempos de retençäo com padrōes conhecidos e a quantificação, pela determinação da área do pico. O aparelho utilizado foi um cromatógrafo a gás da CG Instrumentos Científicos Mod. 37370, equipado com detetor de captura de elétrons, fonte de trítio e coluna 0,3 cm diâmetro x 1,8 m, empacotada com 1,5\% OV-17 + 1,95\% QF-1 em Chromosorb WHP. As condiçöes

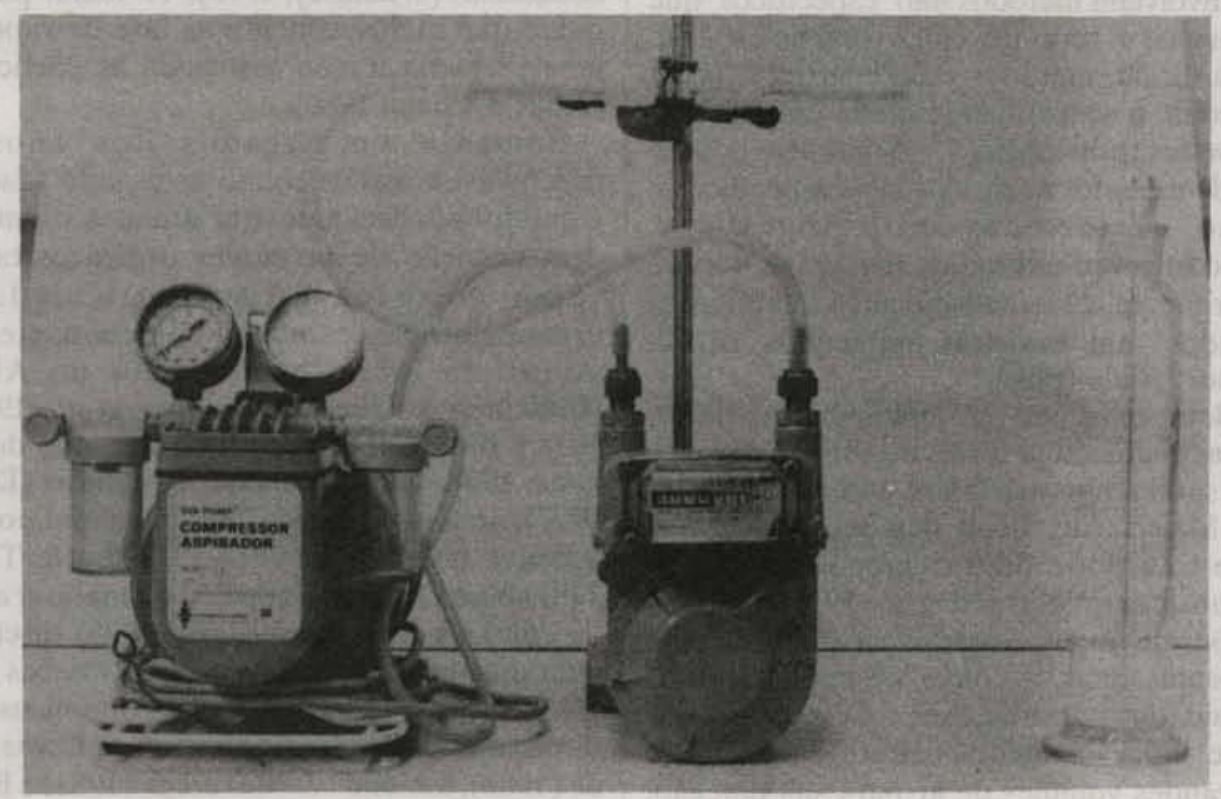

Fig. 1. Amostrador para avaliação da eficiência de coleta de organoclorados no ar. 
cromatográficas foram: temperatura da coluna $=210^{\circ} \mathrm{C}$, temperatura do detetor $=220^{\circ} \mathrm{C}$, temperatura do injetor $=220^{\circ} \mathrm{C}$, volume injetado $=5 \mu \mathrm{l}$. O gás de arraste utilizado foi o Nitrogênio U da Oxigênio do Brasil e a vazão de $20 \mathrm{ml} / \mathrm{min}$.

Para coleta de organoclorados na atmosfera "in situ" utiliza-se um sistema (Fig. 2) que compreende uma bomba de vácuo, um medidor de vazão e um cartucho de aço inox, contendo o adsorvente sólido, previamente purificado.

\section{Resultados e discussão}

A Tabela 1 contém as porcentagens de eficiência de coleta para os adsorventes estudados. Os testes foram feitos em duplicata.

Os compostos organoclorados foram coletados em níveis que correspondem a concentraçōes de 10 a 100 $\mathrm{ng} / \mathrm{m}^{3}$.

A eficiência de coleta variou de 16 a $118 \%$ para o florisil, de 41 a $131 \%$ para a resina XAD-2 e de 64 a $122 \%$ para a espuma de poliuretano.

O florisil apresentou baixa eficiência de coleta, menor que $60 \%$ para a maioria dos compostos analisados, com exceção do $\mathrm{HCB}$, alfa- $\mathrm{HCH}$, gama-HCH, Heptachloro e PCBs, cujos valores variaram entre 73 a $118 \%$.
A resina XAD-2 mostrou-se mais eficiente somente para os organoclorados de baixa e média polaridade, desde o HCB até o Dieldrin (122 a $99 \%$ ).

A espuma de poliuretano apresentou valores abaixo de $100 \%$ com exceção dos PCBs (122\%), entretanto, a eficiência de coleta foi mais uniforme em relaçāo aos compostos de polaridade variável e de pesos moleculares diferentes.

E interessante notar que nenhum dos adsorventes testados retém os compostos mais polares (DDD e DDT) com eficiência superior a $75 \%$.

Outros autores também testaram a eficiência destes adsorventes e diferem dos nossos valores, principalmente devido ao tipo de bomba utilizada que é de menor vazão. Para compostos menos polares, volumes até $300 \mathrm{~m}^{2}$ são suficientes, enquanto que para reter compostos mais polares, volumes entre $1000 \mathrm{e} 2000 \mathrm{~m}^{3}$ são ideais. Lewis \& Jackson (1982) obtiveram EC de $28 \%$ para Aldrin, $89 \%$ para pp'-DDE e $83 \%$ para pp'-DDT, utilizando-se a EPU como adsorvente para um volume de $300 \mathrm{~m}^{3}$. Adams \& Caro (1980), utilizando o mesmo adsorvente, obtiveram valores de $\mathrm{EC}=100 \%$ para $\mathrm{pp}$ '-DDE, $118 \%$ para pp'-DDT e $121 \%$ para Aldrin com volume de $55 \mathrm{~m}^{3}$.

Outros autores, como Billings \& Bidleman (1983), obtiveram valores de EC superiores a $90 \%$ para pp'-DDE e Arochlor 1254 tanto para a resina XAD-2 como para a EPU, utilizando um volume entre $500-700 \mathrm{~m}^{3}$.

Nossos resultados mostram que mesmo utilizando bombas de baixa vazāo, a EPU é a mais indicada para coleta de organoclorados na atmosfera como é a tendência
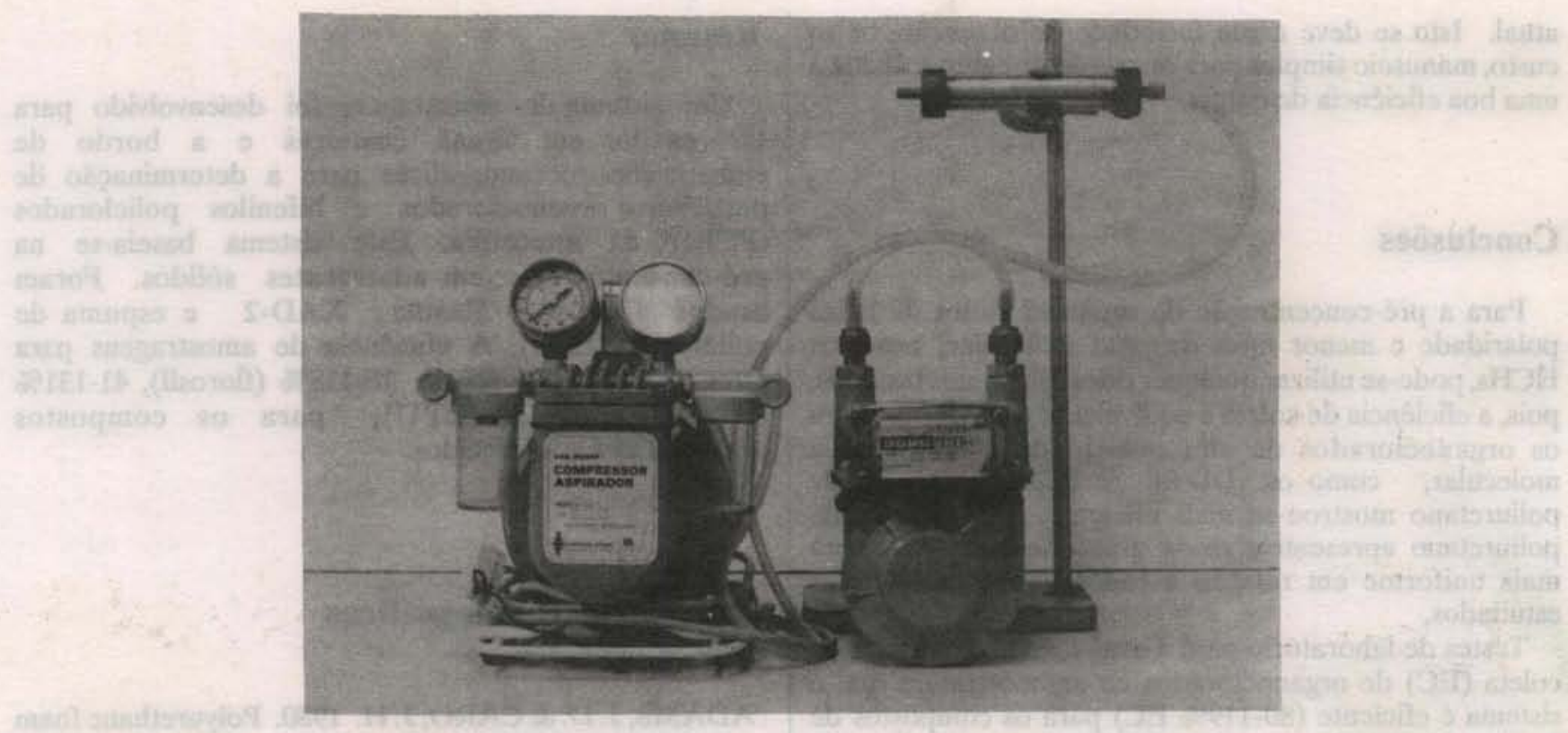

Fig. 2. Sistema para coleta de organoclorados na atmosfera ("in situ"). 
Tabela 1. Eficiência de retenção dos organoclorados da atmosfera nos diferentes adsorventes

\begin{tabular}{|c|c|c|c|c|}
\hline \multirow{2}{*}{ organoclorados } & \multirow{2}{*}{$\begin{array}{l}\text { Quantidade } \\
\text { adicionada, ng }\end{array}$} & \multicolumn{3}{|c|}{ \% Eficiência de coleta } \\
\hline & & Florisil & $X A D-2$ & EPU \\
\hline HСB & 10 & 81 & 122 & 98 \\
\hline a I f a - HCH & 10 & 80 & 118 & 98 \\
\hline gama-HCH & 10 & 118 & 119 & 73 \\
\hline Heptachloro & 10 & 117 & 102 & 71 \\
\hline Aldrin & 20 & 19 & 106 & 86 \\
\hline Hep. Epoxido & 20 & shin zing & 102 & 75 \\
\hline$P P^{\prime}-D D E$ & 30 & 48 & 104 & 76 \\
\hline Dieldrin & 30 & 52 & 99 & 77 \\
\hline$O P^{\prime}-D D D$ & 60 & 30 & 41 & 74 \\
\hline Endrin & IA & 35 & 68 & 66 \\
\hline$O P^{\prime}-D D T$ & $\tan 07 x+5 x$ & 25 & 70 & 66 \\
\hline$P P^{\prime}-D^{\prime} D D$ & 60 & 16 & 45 & 64 \\
\hline$P P^{\prime}-D D T$ & 100 & 20 & 50 & 64 \\
\hline Mirex & 100 & 30 & 56 & 75 \\
\hline Arochlor 1254 & 100 & $\begin{array}{r}73 \\
\end{array}$ & 131 & 122 \\
\hline
\end{tabular}

Volume de ar $=1,0 \mathrm{~m}^{3}$

$E P U=$ espuma de poliuretano

atual. Isto se deve a sua facilidade de obtenção, baixo custo, manuseio simples para operação de campo, aliada a uma boa eficiência de coleta.

\section{Conclusões}

Para a pré-concentração de organoclorados de baixa polaridade e menor faixa de peso molecular, como os HCHs, pode-se utilizar qualquer dos adsorventes testados, pois, a eficiência de coleta é equivalente para os três. Para os organoclorados de alta polaridade e maior peso molecular, como os DDTs e PCBs, a espuma de poliuretano mostrou-se mais eficiente. A espuma de poliuretano apresentou ainda uma eficiência de coleta mais uniforme em relação a todos os organoclorados estudados.

Testes de laboratório para a avaliaçāo da eficiência de coleta (EC) de organoclorados no ar, mostraram que o sistema é eficiente (80-119\% EC) para os compostos de baixa polaridade como os $\mathrm{HCHs}$ e, razoavelmente eficiente (73-131\% EC) para os de alta polaridade, como os PCBs.

\section{Resumo}

Um sistema de amostragem foi desenvolvido para ser usado em áreas costeiras e a bordo de embarcaçōes oceanográficas para a determinação de pesticidas organoclorados e bifenilos policlorados (PCBs) na atmosfera. Este sistema baseia-se na pré-concentração em adsorventes sólidos. Foram usados Florosil, Resina XAD-2 e espuma de poliuretano (EPU). A eficiência de amostragens para estes adsorventes foi de $16-118 \%$ (florosil), $41-131 \%$ (XAD-2) e 66-112 (EPU), para os compostos organoclorados analisados.

\section{Referências bibliográficas}

ADAMS, J.D. \& CARO, J. H. 1980. Polyurethane foam as a trapping agent for airborne pesticides. EPA600/4-80-008. U.S.Environmental Protection Agency. 
ATLAS, E. \& GIAM, C. S. 1986. Sea-air exchange of high-molecular weight synthetic organic compounds. In: Buat- Ménard, P., ed. The role of air-sea exchange in geochemical cycling. Dordrecht, D. Reidel. p. 295-329.

BIDLEMAN, T. F. 1985. High-volume collection of organic vapors using adsorbents. In: Trace analysis. New York, Academic Press. v. 4, p. 51-100.

; CHRISTENSEN, E. J.; BILLINGS, W. N. \& LEONARD, R. 1981. Atmospheric transport of organochlorines in the North Atlantic gyre. J. mar. Res., 39(3):443-464.

\& LEONARD, R. 1982. Aerial transport of pesticides over the northern Indian Ocean and adjacent seas. Atmos. Environ., 16(5):1099-1107.

\& OLNEY, C. E. 1974. Chlorinated hydrocarbons in the Sargasso Sea atmosphere and surface water. Science, N.Y., 183:516-518.

BILLINGS, W. N. \& BIDLEMAN, T. F. 1983. High volume collection of chlorinated hydrocarbons in urban air using three solid adsorbents. Atmos. Environ., $17(2): 383-391$.

DAWSON, R. \& RILEY, J.P. 1977. Chlorine contaning pesticides and polychlorinated biphenyls in British coastal waters. Estuar. coast. mar. Sci., 5(1):55-71.

ENVIRONMENTAL PROTECTION AGENCY (EPA), 1980. Analysis of pesticides residues in human and environmental samples, section $8 \mathrm{~A}, 8 \mathrm{~B}$, Sampling of pesticides in air. EPA-600/8-80-038.

GIAM, C. S.; ATLAS, E. L.; CHAN, H.S. \& NEFF, G. S. 1980. Phthalate esters, PCB and DDT residues in the Gulf of Mexico atmosphere. Atmos. Environ., 14:65-69.

; CHAN, H. S.; NEFF, G. S. \& ATLAS, E. L. 1978. Phthalate esters plasticizers: a new class of marine pollutant. Science, N.Y., 199:419-421.

LAMB, S. I.; PETROWSKI, C.; KAPLAN, I. R. \& SIMONEIT, B. R. I. 1980. Organic compounds in urban atmosphere: a review of distribution, collection, and analysis. J. Air. Pollut. Control Ass., 30:1098.

LEWIS, R. G. 1976. Sampling and analysis of airbone pesticides. In: Lewis, R. G. \& Lee, R. E., eds Air pollution and agriculture process. Cleveland, CRC Press. p. 51.

\& JACKSON, M.D. 1982, Modification and evaluation of a high-volume air sampler for pesticides and semivolatile industrial organic chemicals. Analyt. Chem., 54:592-594.
LEWIS, R. G. \& MacLeod, K. E. 1982. Portable sampler for pesticides and semivolatile industrial organic chemicals in air. Analyt. Chem., 54:310-315.

MONTONE, R. C. 1987. Hidrocarbonetos clorados no litoral do Estado de São Paulo. Dissertação de mestrado. Universidade de São Paulo, Instituto Oceanográfico. 102p.

RICE, C. P.; OLNEY, C. E. \& BIDLEMAN, T. F. 1977. Use of polyurethane foam to collect trace amounts of chlorinated hydrocarbons and other organics form air. Spec. Environ. Rep. Wld. met. Org., (10):216224. WMO-460.

RIGGIN, R. M. 1984. Compendium of methods for the determination of toxic compounds in ambient air. EPA/4-84-041. U.S. Environmental Protection Agency.

SIMONEIT, B. R. T. \& MAZURECK, M. A. 1981. Air pollution: the organic compounds. CRC critical Rev. environ. Control, 11:219.

TANABE, S.; KAWANO, M. \& TATSUKAWA, R. 1982a. Chlorinated hydrocarbons in the Antarctic, Western Pacific and Eastern Indian oceans. Trans. Tokyo Univ. Fish., (5):97-109.

\& TATSUKAWA, R. 1980. Chlorinated hydrocarbonsin the North Pacific and Indian oceans. J. oceanogr. Soc. Japan, 36:217-226.

\&

1983. Chlorinated hydrocarbons in the Southern Ocean. Mem. natn. Inst. polar Res., Spec. issue, 27:64-76.

; KAWANO, M. \& HIDAKA, H. 1982b. Global distribution and atmospheric transport of chlorinated hydrocarbons: $\mathrm{HCH}(\mathrm{BHC})$ isomers and DDT compounds in the Western Pacific, Eastern Indian and Antarctic oceans. J. oceanogr. Soc. Japan, 38:137-148.

VAN DYK, L. P. \& VISWESWARIAH, K. 1975. Pesticides in air: sampling methods. Residue Rev., (55):91-134.

WHEATLEY, G. A. 1973. Pesticides in the atmosphere. In: Edwards, C. A., ed. Environmental pollution by pesticides. London, Plenum Press. p. 365-408.

WOODWELL, G. M.; GRAIG, P. P. \& JOHNSON, H. A. 1971. DDT in the biosphere: where does it go? Science, N.Y., (174):1101-1107.

(Recebido em 16-05-90; aceito em 15-08-91) 